\title{
China CDC in Action - Hypertension Prevention and Control
}

\author{
Lei Hou'; Bo Chen'; Yibing Ji'; Baohua Wang'; Jing Wu ${ }^{1, \text { 1, }}$
}

\section{Summary}

Hypertension contributes to a quarter of all-cause mortality in China. The Chinese government has put forward aims to control and prevent hypertension, and China CDC and other professional public health institutions have carried out a series of related efforts including the promotion of related policies and legislation, the improvement of modifiable risk factors of hypertension in the population, and promoting the National Primary Public Health Services to discover and manage patients with hypertension. China CDC has conducted multiple hypertension-related campaigns such as advocating for salt reduction, weight loss, and increased physical activity. Additionally, on behalf of the Chinese government, China CDC has organized and carried out the construction of national demonstration areas for the comprehensive prevention and control of chronic diseases. As of 2020, 488 national demonstration areas have been established, covering $17.1 \%$ of all counties and districts. In these areas, the lifestyle and health literacy of the population and community-based hypertension management measures have been improved. The Healthy China 2030 calls for a transition from disease treatment to a focus on health, which requires a strategy of hypertension prevention and control that focuses on the whole population rather than on high-risk populations. The Primary Health Care, Medicine and Health Promotion Law was officially implemented on June 1, 2020 and provides the legal basis for this strategy change in the prevention and control of hypertension. Ongoing public health legislation should involve non-communicable diseases such as hypertension in addition to communicable diseases.

There are 270 million patients with hypertension in China. The Global Burden of Disease (GBD) showed that hypertension had become the primary contributor to disability-adjusted life years in China, contributing to $24.6 \%$ of all-cause mortality (1-2). The proportion of lost life years from an early death caused by hypertension in cardiovascular and cerebrovascular disease is $64.5 \%$ and $72.8 \%$, respectively, ranking first among all risk factors of cardiovascular and cerebrovascular diseases (2). The Healthy China Initiative (2019-2030), which was initiated by the Chinese government in 2019, has established goals for hypertension prevention and control such as regular blood pressure (BP) monitoring for adults aged 18 years or older, increasing awareness rates, and normalizing the management, treatment, and control of hypertension (Table 1). Professional public health institutions, including China CDC, attach great importance to this public health issue, and much work has been conducted in this area. This study aimed to explore the challenges and opportunities of hypertension prevention and control by reviewing the work of the China CDC in changing strategies, lifestyle interventions, and patient management.

\section{CREATING A SUPPORTIVE ENVIRONMENT FOR CONTROLLING AND PREVENTING HYPERTENSION}

\section{Policy Making and Legislation}

The prevention and control of hypertension is key to achieving the strategic goal of reducing premature mortality from major chronic diseases by $30 \%$ compared with 2015 and increasing the life expectancy per capita in the next 10 years. China has established many policies for hypertension prevention and control (3). In 2016, the Healthy China 2030 calls for a transition from disease treatment to a focus on health. In 2018, China CDC suggested that the prevention and control of hypertension must change from a highrisk population strategy led by the health department to a population-wide strategy that includes all parts of society (3); risk factors of hypertension such as high salt intake, overweight or obesity, and insufficient exercise should be addressed while strengthening screening and management for hypertension. This perspective on practicing the population-wide strategy of hypertension has been supported by the 2020 joint 
TABLE 1. Goals of hypertension prevention and control in Healthy China Initiative.

\begin{tabular}{lccc}
\hline \multicolumn{1}{c}{ Indicators (\%) } & Baseline & In 2022 & In 2030 \\
\hline Awareness rate in residents aged 30 years or older & 47 (in 2012) & $\geq 55$ & $\geq 65$ \\
Normalized management rate in patients managed & 50 (in 2015) & $\geq 60$ & Rising \\
Treatment rate in patients & 41.1 (in 2012) & Rising & Rising \\
Control rate in patients & 13.8 (in 2012) & Ring \\
\hline
\end{tabular}

position statement from the World Stroke Organization and the World Heart Federation (4). The Primary Health Care, Medicine and Health Promotion Law, officially implemented on June 1, 2020, has established health promotion as a legal provision for the first time in China (5). This legislation provides the legal foundation for changing the hypertension prevention and control strategy from one focusing on high-risk populations to a strategy that includes the whole population.

\section{Establishment of National Demonstration}

Areas for the Comprehensive Prevention and Control of Chronic Diseases

The prevention and control of hypertension requires participation from the whole of society. Since 2010, China CDC has organized and carried out construction of national demonstration areas (whole counties or districts) for the comprehensive prevention and control of chronic diseases. The demonstration areas were designed on the principles of a governmentled program with departmental cooperation, mobilization of society, and the participation of all residents. In these areas, the government implemented measures to support health, promote a healthy lifestyle, and improve the health literacy of the residents in ways that were closely associated with reducing hypertension. As of 2020, China has established 488 national demonstration areas covering $17.1 \%$ of all counties and districts. A study on the effectiveness of implementation indicated residents living in the national demonstration areas with implementation scores of higher than $50 \%$ were more likely to be aware of relevant knowledge regarding salt reduction (odds ratio (OR) 1.352, 95\% confidence interval (CI): 1.151-1.589), oil reduction (OR 1.477, 95\% CI: 1.249-1.746), and recommendations on physical activity (OR 1.975, 95\% CI: 1.623-2.403) in comparison to those with $50 \%$ or lower implementation scores (G). The normalized management rate of patients with hypertension, defined as percent of achieving management standards in patients managed within one year, in the national demonstration areas was found to be $62.1 \%(\sigma)$.

\section{IMPROVEMENT IN THE MODIFIABLE RISK FACTORS OF HYPERTENSION IN THE WHOLE POPULATION}

In 2007, China CDC and the National Patriotic Health Campaign Office launched a national activity - China Healthy Lifestyle for All - focusing on "ten thousand steps a day and a balance between eating and moving". In 2016, the Healthy China strategy incorporated the goals of salt, oil, and sugar reduction and included a focus on oral, weight, and bone health. Salt reduction and weight loss are of particular importance for the prevention and control of hypertension.

High sodium intake is the leading risk factor of hypertension. The World Health Organization (WHO) recommends a 30\% reduction in salt intake by 2025 , with an eventual target of less than 5 grams per day worldwide. Beginning in 2011, a 5-year population-based intervention to reduce sodium consumption has been conducted in Shandong Province, which has nearly 100 million people. The intervention is the Shandong Ministry of Health Action on Salt and Hypertension (SMASH) and includes health education, the distribution of saltrestriction spoons, and promotion of low-sodium products. A comparative analysis between two crosssectional surveys from the SMASH program showed that 24-hour mean urinary sodium excretion among adults decreased by $24.8 \%$, potassium excretion increased by $15.1 \%$, and the $\mathrm{Na}^{+} / \mathrm{K}^{+}$ratio decreased by $36.6 \%$ (7). Another 3-year cohort study from the SMASH program firstly indicated that using a saltrestriction spoon when cooking was associated with reduced salt intake, which slowed BP deterioration in the real world. Particularly, individuals using a 2-gram salt-restriction spoon showed significant decreases in the mean 24 -hour urinary $\mathrm{Na}^{+} / \mathrm{K}^{+}$ratio in comparison with those who did not use a salt-restriction spoon $(-3.49$ vs. -2.22$)$ (8). China CDC is currently sharing the SMASH experience with many provinces 
across China.

Overweight and obesity are associated with many chronic noncommunicable diseases, such as hypertension and diabetes, and are linked to a poor diet and lack of physical activity. Losing weight has been listed as a key component of managing patients with hypertension or diabetes, along with health education provided by primary healthcare sites and local CDCs. Weight loss has also been incorporated into the Rational Diet Action and National Fitness Action of Healthy China Initiative with the aim of slowing the increase of the adult obesity rate. In 2020, a national program of obesity prevention and control, particularly implemented in children and adolescents, is being promoted. Currently, China Healthy Lifestyle for All is the most popular weight-loss campaign in China and promotes maintaining a healthy weight by reducing oil and sugar intake and increasing sports activities.

\section{SCREENING AND MANAGEMENT OF PATIENTS WITH HYPERTENSION}

In 2009, China implemented the National Primary Public Health Services (NPPHS) program, which considerably affects the Chinese public health system. This program provided several free primary healthcare services for all Chinese people, regardless of age, sex, ethnic group, residence, occupation, or income. The funding for the NPPHS was supported by the annual central budget and covered more than 1.3 billion people. The standard budget subsidy gradually increased from $15 \mathrm{RMB}$ per person per year in 2009 to $74 \mathrm{RMB}$ in 2020.

The screening and management of patients with hypertension - a key part of the NPPHS — includes BP measurement at least once a year for residents aged 35 years and over, 4 face-to-face follow-ups (including symptom assessment, check-ups such as measurement of body mass index, and lifestyle intervention), 1 comprehensive physical examination per year for each patient with hypertension, medication guidance, and referral services. These services are provided by community-based medical institutions such as primary hospitals, health centers, and clinics that are supervised by health administrative departments and are guided by the CDC and public hospitals. The NPPHS managed approximately 100 million patients with hypertension in 2020, and this number is set to increase to 110 million by 2025 (9).

\section{CHALLENGES AND OPPORTUNITIES}

There are approximately 170 million patients with hypertension not covered by the BP management service from the NPPHS so more challenges await China CDC. We should take advantage of the new health promotion law to promote primary hypertension prevention measures in the whole population, such as reducing salt intake, controlling body weight, and increasing physical activity. Furthermore, following the theme of Hypertension Day (October 8 since 1998) — "All Persons 18 Years Old and Above Should Know Their Blood Pressure" - put forward by the National Health Commission of China in 2019, population-wide BP screening policies should be rigorously implemented, including BP measurements at patients' first visits to outpatient clinics, provision of physical examinations for the working population, promotion of health in schools, and instituting an NPPHS documentation service, and considering that more than a half of patients with hypertension do not know their condition with this disease in China (10). Next, China CDC and local CDCs need to participate in more NPPHS work such as guidance, supervision, and assessment for the NPPHS and, particularly, allow more patients and more scientific service programming to be involved in the NPPHS. Nevertheless, strengthening public health measures and primary healthcare will result in the effective population-based management of hypertension that would eventually reduce the total cardiovascular risk of this population.

Presently, there was still a gap between legislation and practice for the prevention and control of noncommunicable diseases (NCDs), such as hypertension, in China. For example, the orientation of paying more attention to medicine than prevention has not been fundamentally reversed through legislation (5). The status and power of CDCs needs to be further elevated by improving legislation. Public health physicians should be given the right of prescribing essential drugs in primary healthcare to enhance their ability to participate in the NPPHS and promote the coordination of medicine and prevention $(3,5)$. NCD prevention and control, which is the cornerstone of Healthy China Strategy, is being challenged by the coronavirus disease 2019 (COVID-19) pandemic worldwide, and ongoing public health legislation should not only include communicable diseases but also involve NCDs. In addition, China CDC is currently set to establish a scientific research institution 
whose staff will mostly be scientific researchers. The new health promotion law defines China CDC as a professional public health institutions, which is included in medical and healthcare institutions, but excludes scientific researchers from the definition of medical staff. This limits the public health practice capacity, including hypertension prevention and control, of China CDC and its staff. Nevertheless, location on both medical and healthcare and scientific research are factually essential for the China CDC and its staff. A fundamental change in the system of disease control and prevention is coming following the pandemic, and this change may give a chance to increase the role of China CDC for hypertension prevention and control.

Conflicts of interest: No conflicts of interest were reported.

doi: $10.46234 / \mathrm{ccdcw} 2020.212$

\# Corresponding author: Jing Wu, wujing@chinacdc.cn.

${ }^{1}$ National Center for Chronic and Noncommunicable Disease Control and Prevention, Chinese Center for Disease Control and Prevention, Beijing, China.

Submitted: August 07, 2020; Accepted: September 16, 2020

\section{REFERENCES}

1. Zhou MG, Wang HD, Zeng XY, Yin P, Zhu J, Chen WQ, et al. Mortality, morbidity, and risk factors in China and its provinces, 1990 2017: a systematic analysis for the global burden of disease study 2017. Lancet 2019;394(10204):1145 - 58. http://dx.doi.org/10.1016/S0140-
6736(19)30427-1.

2. Liu MP, Li YC, Liu SW, Wang W, Zhou MG. Burden on bloodpressure-related diseases among the Chinese population, in 2010. Chin J Epidemiol 2014;35(6):680 - 3. http://dx.doi.org/10.3760/cma.j.issn. 0254-6450.2014.06.014. (In Chinese).

3. Hou L. Prevention and control of hypertension in the background of healthy China. Natl Med J China 2018;98(39):3134-7. http://dx.doi.org/10.3760/cma.j.issn.0376-2491.2018.39.002. (In Chinese).

4. Brainin M, Sliwa K. WSO and WHF joint position statement on population-wide prevention strategies. Lancet 2020;396(10250): 533-4. https://www.thelancet.com/journals/lancet/article/PIIS0140-6736 (20)31752-9/fulltext.

5. Hou L. Tamping legal basis of "Health First, Prevention First": deliberation and advices on the primary health care, medicine and health promotion law. Chin J Soc Med 2020;37(3): 238-41. http://d. wanfangdata.com.cn/periodical/gwyx-shyx202003004. (In Chinese).

6. Zhang J, Jin RR, Li JJ, Li JL, Su XW, Deng GJ, et al. Study on the effectiveness of implementation: the national demonstration areas for comprehensive prevention and control of non-communicable diseases. Chin J Epidemiol 2018;39(4):394 - 400. http://dx.doi.org/10.3760/ cma.j.issn.0254-6450.2018.04.002. (In Chinese).

7. Xu AQ, Ma JX, Guo XL, Wang LH, Wu J, Zhang JY, et al. Association of a province-wide intervention with salt intake and hypertension in Shandong province, China, 2011-2016. JAMA Intern Med 2020; 180(6):877 - 86. http://dx.doi.org/10.1001/jamainternmed.2020.0904.

8. Hou L, Guo XL, Zhang JY, Chen X, Yan LX, Cai XN, et al. Associations between salt-restriction spoons and long-term changes in urinary $\mathrm{Na}^{+} / \mathrm{K}^{+}$ratios and blood pressure: findings from a populationbased cohort. J Am Heart Assoc 2020;9(14):e014897. http://dx.doi.org/ 10.1161/JAHA.119.014897.

9. Hou L, Chen XR, Chen B, Liu LJ, Sun XH, Zou YW, et al. Pharmacological therapy and blood pressure control in primary health care sites in China: data from 254,848 hypertensive patients. Clin Epidemiol 2018;10:1467 - 78. http://dx.doi.org/10.2147/CLEP.S172 567.

10. Li YC, Yang L, Wang LM, Zhang M, Huang ZJ, Deng Q, et al. Burden of hypertension in China: a nationally representative survey of 174,621 adults. Int J Cardiol 2017;227:516 - 23. http://dx.doi.org/10.1016/ j.ijcard.2016.10.110. 